\title{
The Impact of Incretin-Based Medications on Lipid Metabolism
}

\author{
Habib Yaribeygi $\mathbb{D}^{1}{ }^{1}$ Mina Maleki, ${ }^{2}$ Alexandra E. Butler, ${ }^{3}$ Tannaz Jamialahmadi, ${ }^{4,5}$ \\ and Amirhossein Sahebkar iD $6,7,8$ \\ ${ }^{1}$ Research Center of Physiology, Semnan University of Medical Sciences, Semnan, Iran \\ ${ }^{2}$ Urology and Nephrology Research Center, Shahid Beheshti University of Medical Sciences, Tehran, Iran \\ ${ }^{3}$ Research Department, Royal College of Surgeons in Ireland, PO Box 15503, Adliya, Bahrain \\ ${ }^{4}$ Department of Food Science and Technology, Quchan Branch, Islamic Azad University, Quchan, Iran \\ ${ }^{5}$ Department of Nutrition, Faculty of Medicine, Mashhad University of Medical Sciences, Mashhad, Iran \\ ${ }^{6}$ Applied Biomedical Research Center, Mashhad University of Medical Sciences, Mashhad, Iran \\ ${ }^{7}$ Biotechnology Research Center, Pharmaceutical Technology Institute, Mashhad University of Medical Sciences, Mashhad, Iran \\ ${ }^{8}$ Department of Biotechnology, School of Pharmacy, Mashhad University of Medical Sciences, Mashhad, Iran
}

Correspondence should be addressed to Habib Yaribeygi; habib.yari@yahoo.com

and Amirhossein Sahebkar; amir_saheb2000@yahoo.com

Received 30 September 2021; Accepted 6 December 2021; Published 29 December 2021

Academic Editor: Toshiyasu Sasaoka

Copyright (c) 2021 Habib Yaribeygi et al. This is an open access article distributed under the Creative Commons Attribution License, which permits unrestricted use, distribution, and reproduction in any medium, provided the original work is properly cited.

\begin{abstract}
Pathophysiological pathways that are induced by chronic hyperglycemia negatively impact lipid metabolism. Thus, diabetes is commonly accompanied by varying degrees of dyslipidemia which is itself a major risk factor for further macro- and microvascular diabetes complications such as atherosclerosis and nephropathy. Therefore, normalizing lipid metabolism is an attractive goal for therapy in patients with diabetes. Incretin-based medications are a novel group of antidiabetic agents with potent hypoglycemic effects. While the impact of incretins on glucose metabolism is clear, recent evidence indicates their positive modulatory roles on various aspects of lipid metabolism. Therefore, incretins may offer additional beneficial effects beyond that of glucose normalization. In the current review, how these antidiabetic medications can regulate lipid homeostasis and the possible cellular pathways involved are discussed, incorporating related clinical evidence about incretin effects on lipid homeostasis.
\end{abstract}

\section{Introduction}

Diabetes mellitus (DM) is an increasingly prevalent metabolic disorder, the major sign and symptom being hyperglycemia and polyuria, respectively [1]. This chronic disease is a major underlying cause for severe debilitating conditions such as cardiovascular disorders and renal failure [2]. Aberrations in the normal physiological metabolic pathways of most substrates, including lipids, are present in DM, and the disordered metabolism can induce the onset and progression of metabolic disorders [3-5]. Many diabetic complications involve dyslipidemia, and lipid homeostasis has obvious impacts on the function of most organs, important examples being the kidneys, heart, blood vessels, the neuro- nal network, and testes [6]. Therefore, normalizing lipid metabolism in the diabetic milieu is an important goal for prevention of diabetes-induced complications $[3,6]$.

Incretin-based medications are a new class of antidiabetic drugs that effectively lower circulating glucose, acting through various cellular pathways [7]. These antihyperglycemic agents have significant effects on body metabolism and increase insulin sensitivity via multiple molecular mechanisms [7]. Although some evidence indicates beneficial effects of these drugs on adipocytes and lipids [8], their exact role in lipid homeostasis is, to date, poorly understood. Should incretins be able to normalize lipid homeostasis, this would extend their therapeutic effects beyond their hypoglycemic role in diabetic patients. Therefore, in the current 
study, we present an update on current knowledge regarding the impact of incretins on lipid homeostasis.

\section{Incretin-Based Antidiabetic Drugs}

Incretins are a family of intestinal hormones that includes glucagon-like peptide-1 (GLP-1) and gastric inhibitory peptide (GIP) and that exerts their antidiabetic effects via diverse mechanisms such as glucagon release inhibition, stimulating insulin secretion, causing delay in gastric emptying and appetite suppression, reducing the absorption of intestinal nutrients, improving lipid metabolism, and promoting pancreatic $\beta$-cell function (Figure 1) [9-13]. GLP-1 is mainly secreted by intestinal enteroendocrine L-cells (as well as certain neurons within the nucleus of the solitary tract), while GIP is synthesized in the gastrointestinal tract by $\mathrm{K}$ cells located in the mucosa of the duodenum and jejunum [9-13]. The GLP-1 peptide acts by binding to its specific receptor, the GLP-1 receptor (GLP-1R), that is mainly located on pancreatic $\beta$-cells $[11,14]$. GLP-1 binding to GLP-1R is followed by an increase in production of cyclic adenosine monophosphate (cAMP), cellular depolarization, and intracellular calcium augmentation, leading to insulin secretion from pancreatic $\beta$-cells $[11,14]$.

Two main classes of incretin antidiabetic drugs have been developed, the GLP-1 receptor agonists (GLP-1RAs) and dipeptidyl peptidase-4 inhibitors (DPP-4is) (Table 1) $[9,15]$. Besides antidiabetic actions, incretin-based drugs have a plethora of beneficial effects on body organs [16-23]. GLP-1RAs reduce blood glucose by stimulating glucose-dependent insulin release from the pancreatic islets, while DPP-4i increase the circulatory level of endogenous GLP-1 by inhibiting the protease enzyme DPP-4, a serine exopeptidase which is physiologically responsible for GLP1 metabolism and inactivation [9, 15]. DPP-4 inhibitors and GLP-1RA have similar hypoglycemic effects, although they may have some differences in pharmacological effects such as influence on body weight and risk of adverse effects [24] (Table 1).

\section{Lipids, Physiology, and Metabolism}

Lipids are hydrocarbonated micromolecules that are not soluble in water but can be dissolved in nonpolar solvents [26]. Due to differences in structure and function, there are numerous types of lipids: triglycerides (TG), phospholipids (PL), cholesterol (CLS), and lipidemic molecules such as sphingolipids, glycolipids, and prostaglandins [27]. TGs and the PLs consist of free fatty acids (FFA) (simple longchain hydrocarbon organic acids with the common formula $\mathrm{C}_{\mathrm{N}} \mathrm{H}_{\mathrm{N}} \mathrm{COOH}$ ), whilst CLS has no complete fatty acid in its structure [27]. However, CLS has many of the biochemical properties of the lipids since its nucleus is made of fatty acid-like biomolecules [27]. Lipids have many physiological functions, including energy storage, signaling activities, and structural functions [27]. Lipids are incorporated into the eukaryotic cell membrane structure by forming a double layer membrane known as a lipid bilayer [27]. They are also involved in the structure of many steroids and steroid hor-

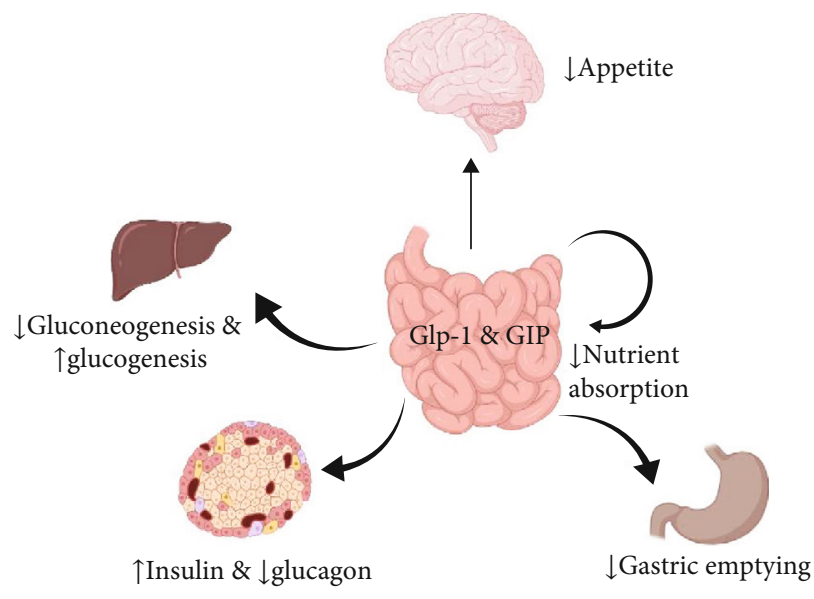

FIGURE 1: The major metabolic effects of incretins.

mones such as vitamin $\mathrm{D}_{3}$, prostaglandins, sex hormones, and adrenal steroids (glucocorticoids and mineralocorticoids) [27]. Therefore, lipids and their derivatives are closely involved in homeostasis and are vital for human health.

Adipose tissue (AT), a metabolic organ involved in energy homeostasis in the body, consists of fat that is largely composed of TGs and PLs $[26,28]$. Originally considered to be inert tissue formed from storage of excess energy, AT was later shown to have important biological function, synthesizing biomolecules such as adipokines and adiponectins and releasing them into the circulation [28]. These peptides have significant hormonal effects on most metabolic pathways, and hence, $\mathrm{AT}$ is now recognized as an endocrine organ with significant metabolic impacts [6].

Lipid metabolism encompasses the processes of absorption, synthesis, polymerization, conversion, and degradation of the lipid molecules [26]. These processes are finely tuned, exhibiting a delicate dynamic equilibrium (in the healthy physiologic state) which ultimately determines the total fat mass of the body [26]. While some types of lipids are constantly being oxidized to provide for the metabolic needs for the body, others are being synthesized, replaced, and stored $[26,29]$. Lipid metabolism is under the influence of many endogenous and exogenous factors [29] and is regulated by hormones such as growth hormone, sex steroids, adipokines, adrenal steroids, and thyroid hormones as well as neuronal stimuli [30]. Many physiological, pathological, and social stimuli, such as exercise, the intensity of physical activity, feeding habits, and stressors, are able to modulate lipid metabolism [30].

\section{The Importance of Lipid Homeostasis in Health and Disease}

Lipid homeostasis is critical for the function of most physiologic systems, examples being the cardiovascular system, kidneys, retina, and nervous system, and is therefore important for overall physical health [31]. A strong body of evidence links dyslipidemia to many life-threatening disorders such as atherosclerosis, nephropathy, fatty liver disease, and thrombosis $[31,32]$. For example, hypercholesterolemia 
TABLe 1: The two main classes of incretin-based antidiabetic drugs.

\begin{tabular}{|c|c|c|c|}
\hline Classes & Approved forms & Mechanisms of action & Ref. \\
\hline GLP-1RA & Exenatide (exendin-4), Albiglutide, liraglutide, lixisenatide, semaglutide, dulaglutide & Agonists of intrinsic incretins & {$[9,15]$} \\
\hline DPP-4i & Sitagliptin, saxagliptin, vildagliptin, linagliptin & Inhibit incretin inactivation & {$[24,25]$} \\
\hline
\end{tabular}

TABLE 2: The impact of incretin-based medications on lipid metabolism.

\begin{tabular}{|c|c|c|c|}
\hline Lipid metabolism & Effects of incretin-based therapy & Ref. & $\begin{array}{c}\text { Clinical } \\
\text { evidence }\end{array}$ \\
\hline $\begin{array}{l}\text { Lipogenesis and } \\
\text { lipolysis }\end{array}$ & Reduces lipogenesis mainly thru an AMPK-dependent pathway & $\begin{array}{c}{[57-61,63,} \\
74]\end{array}$ & $\begin{array}{l}{[63,70,107} \\
108]\end{array}$ \\
\hline Lipid peroxidation & Inhibits lipid peroxidation and reduces lipotoxicity & $\begin{array}{c}{[21,80-82,} \\
84]\end{array}$ & {$[88]$} \\
\hline Fatty acid oxidation & Induces and promotes fatty acid oxidation & {$[49,95]$} & [93] \\
\hline Cholesterol synthesis & $\begin{array}{l}\text { Inhibits HMG-CoA reductase and cholesterol biosynthesis, reduces atheroma } \\
\text { plaque formation }\end{array}$ & $\begin{array}{l}{[98,100} \\
101]\end{array}$ & {$[102]$} \\
\hline Lipid absorption & Commonly reduces intestinal lipid absorption & {$[103,104]$} & {$[105]$} \\
\hline
\end{tabular}

is an underlying cause of atheroma plaques, atherosclerosis, and myocardial infarctions [33]. Dyslipidemia also negatively impacts renal sufficiency [34] and retinal functions $[35,36]$. In the diabetic state, conditions are more conducive for dyslipidemia and dysregulated fat metabolism [32], and it is likely that all diabetic complications are in some ways associated with dyslipidemia [37, 38]. Dyslipidemia can precipitate and progress diabetic complications via several pathologic pathways [38]. Therefore, the majority of patients with diabetes are prescribed lipid-modulating drugs in addition to antidiabetic agents to improve lipid homeostasis and help prevent the onset of diabetic complications [39].

\section{Incretins and Lipid Homeostasis}

Beyond their hypoglycemic effects, incretin-based drugs exert modulatory effects on various steps of lipid metabolism $[40,41]$ (Table 2). These effects can help diabetic patients to normalize fat metabolism whilst concurrently normalizing glycemic levels [39]. In the following sections, we will discuss these metabolic effects.

5.1. Lipogenesis and Lipolysis. Lipolysis and lipogenesis are the two main metabolic processes in lipid metabolism [42]. They are key determinants of adiposity, and the amount of stored lipids is achieved by promoting or reducing lipid reserves in adipocytes [43]. Lipogenesis is the metabolic process in which FFA and TG are synthesized from different substrates, such as carbohydrate, acetyl-coenzyme A (CoA), and glycerol [44]. FFAs are synthesized in the cytoplasm of the cells (specifically the mitochondria) from acetyl-CoA (in a process known as the de novo pathway), whilst TG synthesis takes place mainly in the membrane of the smooth endoplasmic reticulum (SER) [44]. Both processes occur primarily in the liver and adipose tissue, but in individuals with a high caloric diet and where there is high carbohydrate availability, adipose tissue functions as the major site of lipogenesis [44]. However, other tissues, such as the kidneys, brain, lung, and gut, may also produce lipids to some extent [45]. Lipogenesis is a highly controlled metabolic process [46, 47] and is influenced by both stimulatory and inhibitory factors such as transcriptional elements, hormones, and metabolic enzymes [47]. Uncontrolled or pathological lipogenesis is directly linked to metabolic disorders and obesity, and therefore, many lipogenesismodulating pharmacologic agents have been developed [48].

Lipolysis (or the degradation of lipid molecules into their constituent parts) is the other key lipid metabolic process which uniquely occurs in white adipocyte tissue [43]. In this process, TGs break down into their constituent molecules, as FFAs and glycerol, via hydrolysis [43]. Lipolysis takes place mainly at the surface of cytosolic lipid droplets in adipocytes and releases the FFAs and glycerol for use by other tissues [43]. During fasting, lipolysis is induced in order to supply the required FFAs for oxidative metabolism [49]. Lipolysis also prevents serum FFA increase which may result in lipotoxicity [50]. As with lipogenesis, the lipolytic process is under the influence of many stimuli and imbalances impacting lipid metabolism may induce a wide array of metabolic disorders such as obesity, insulin resistance, atherosclerosis, nonalcoholic fatty liver disease (NAFLD) and DM [51]. Therefore, the appropriate lipolytic balance is of great importance in diabetes to prevent downstream complications [51].

Incretin-based drugs are able to modulate both lipogenesis and lipolysis [42]. Sancho et al., in an in vivo study, found that GLP-1 impacts kinases, such as PI3K, p44, and p42 MAPKs and possibly PKC, that are involved in lipolytic and lipogenic processes [42]. They found that GLP-1 and its agonists greatly impact lipogenesis and lipolysis in rat adipocytes [42]. Recent evidence confirms that GLP-1 interacts with the major lipid metabolic enzymes such as lipase [52, 53], pyruvate dehydrogenase [54], acetyl-CoA carboxylase $[55,56]$, and fatty acid synthase [57].

Ben-Shlomo and colleagues in 2011 demonstrated that GLP-1 inhibits lipogenesis via an AMPK-dependent 
pathway in high fat diet (HFD) rats [58]. They found that GLP-1 therapy suppressed lipogenic enzymes such as sterol response element binding protein-1c (SREBP-1c), stearoyl CoA desaturase-1 (SCD-1), fatty acid synthase (FAS), and carnitine palmitoyl transferase-1 (CPT-1) in hepatic cells of experimental rats [58]. Parlevliet et al. in 2012 provided further evidence demonstrating that both GLP-1RA (CNTO3649) and DPP-4i (exendin-4) decrease hepatic lipogenesis in HFD mice [59]. They found that GLP-1 therapy downregulates the genes involved in lipogenesis such as SREBP-1c, FAS, diacylglycerol O-acyltransferase 1 (Dgat1), and apolipoprotein B synthesis (ApoB) [59]. Moreover, Ideta and coworkers in 2015 reported that the DPP-4i teneligliptin reduces hepatic lipogenesis by an AMPK-dependent process in mice [60]. They showed that teneligliptin therapy activates the AMPK pathway and attenuates expression of lipogenic genes to improve NAFLD in a mouse model [60]. A signaling axis between GLP-1 and lipogenesis was suggested by more studies in which incretin-based medications ameliorate lipogenesis via AMPK activation pathway $[57,61]$. GLP-1 therapy may also increase postprandial chylomicron synthesis, thereby increasing fat storage and normalizing serum lipid levels [62]. The weight of evidence therefore suggests that GLP-1 therapy is promising for modulation of lipogenesis in diabetic patients [63].

Incretin-based medications may also be able to modulate lipolysis [64]. GLP-1 agonists reduce adiposity by induction of lipolysis [65]. The first evidence for this was reported by Ruiz-Grande et al. in 1992 [66]. They found that GLP-1 [1-36] exerts lipolytic effects on cultured adipocytes of rats via stimulating different receptors than for the glucagon hormone [66]. Yaney et al. in 2001 demonstrated that GLP-1 induces lipolysis and releases FFAs in a cAMP-dependent manner in clonal pancreatic beta cells [67]. In another study, GLP-1 was able to induce lipolysis only at high doses in human isolated adipocytes [68]. In this study, GLP-1 exerted dual effects on lipid metabolism via inhibition of lipolysis and promotion of lipogenic pathways at a low concentration [68]. $\mathrm{Xu}$ and colleagues in 2016 provided further evidence demonstrating that exendin-4 promoted lipolysis via potentiation of antioxidative defenses in 3T3-L1 adipocytes [49]. They found that exendin-4 increases phosphorylated hormone-sensitive lipase (HSL), a major hallmark of lipolysis [49]. Other evidence by Patel et al. in 2017 established that a synthesized agonist of GLP-1R (known as Aib2 C24 chimera) controlled lipolysis and modulated dyslipidemia in obese hamsters [69]. Recent clinical evidence from 2019 reported that liraglutide, a GLP-1RA, reduced adiposity and body fat mass by promoting lipolysis in obese patients with type 2 diabetes mellitus (T2DM) [70]. More recent evidence presented by Pereira et al. indicated that GLP-1R induction increases lipolysis and reduces adiposity in human adipocytes [71]. A study by Rago and coworkers in 2020 reported that human sperm possess GLP-1R that enables them to control lipid metabolism via lipogenic and lipolytic pathways [72], a finding that emphasizes the role of these receptors in lipid homeostasis [72]. Thus, incretin-based therapies could be promising agents for improving dyslipidemia and lipid homeostasis [73] by altering the balance between lipogenesis and lipolysis toward lower adiposity and prevention of diabetes-related dyslipidemia-induced complications such as diabetic nephropathy $[74,75]$ and beta cell dysfunction [76].

5.2. Lipid-Peroxidation/Lipotoxicity. Lipid peroxidation is an injurious cellular event caused by oxidative degradation of lipids in which free radical species procure electrons from cell membrane lipids and, in turn, produce toxic byproducts such as lipid hydroxides [77]. Different forms of these byproducts, such as malondialdehyde (MDA), F2-isoprostanes, and 4-hydroxynonenal (HNE), are increased in oxidative stress and are therefore recognized as biomarkers of oxidative damage [77]. These byproducts are also able to bind to DNA at specific points and promote mutations by forming DNA adducts, thereby producing additional biomarkers such as 8-oxo-2' -deoxyguanosine (8oxodG) [78]. These events most commonly occur in environments with weakened antioxidant capacity such as diabetes [79].

GLP-1 mimetics have varying abilities to protect against lipid peroxidation [80]. These drugs have potent direct and indirect antioxidative capacities (such as antioxidant defense system (ADS) potentiation, prooxidant inhibition, steroid receptor coactivator (SRC) protein suppression, and improvement in mitochondrial function) that enable them to prevent oxidative stress-induced lipid peroxidation [21]. Patel and colleagues in 2013 reported that GLP-1 therapy prevented lipid peroxidation via improving oxidative stress in mice [80]. Another study reported that exenatide reduced MDA content (a marker of lipid peroxidation) in patients with T2DM [81]. A recent article confirmed these reports and demonstrated that the GLP-1 mimetic, myricetin (a novel DPP-4i), reduced lipid peroxidation in the oxidative milieu of diabetic mice [82]. Therefore, clear evidence indicates that incretin-based therapies inhibit lipid peroxidation via increasing $\mathrm{ADS}$ potency and reducing oxidative stress.

GLP-1 mimetics have also been suggested as key therapeutic agents for prevention of lipotoxicity [83], functioning through several pathways (Figure 2). These drugs combat excess serum lipid levels through their potent antiinflammatory and antioxidative actions [83]. Huang and coworkers in 2015 demonstrated that liraglutide mitigates inflammation and oxidative stress and promotes beta cell proliferation and improved islet function [83]. Armstrong et al. in 2016 reported that liraglutide inhibits lipotoxicity in patients with NAFLD by increasing insulin sensitivity in adipocytes and improving liver function [84]. Gu et al. in 2016 demonstrated that exendin-4 attenuates lipotoxicity through ERK1/2 activation and improvements in mitochondrial function [85]. Another proposed mechanism of action, as reported by Somm et al. in 2021 in mice, is through prevention of ceramide accumulation [86]. Liu et al. in 2020 reported that GLP-1 therapy reduces lipotoxicity by suppression of the inflammatory mediator NF- $\kappa \mathrm{b}$ in mice [87].

It has also been suggested that impaired GLP-1 secretion is associated with dyslipidemia (hypertriglyceridemia) and the degree of lipotoxicity [88]. Wang and colleagues in 2018 found that diabetic patients with dyslipidemia have impaired GLP-1 secretion that directly correlated with 


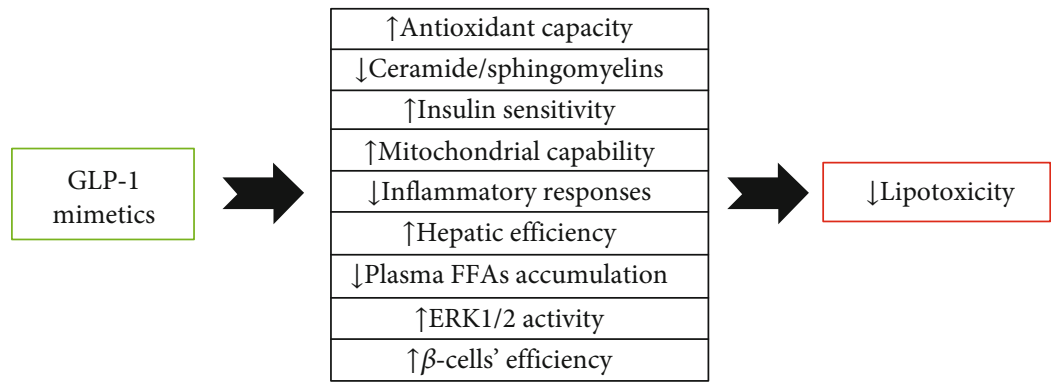

FIgURE 2: The antilipotoxicity effects of GLP-1 mimetics.

lipotoxicity [88]. Moreover, GLP-1 secretion may be increased in response to lipotoxicity and dyslipidemia in an effort to mitigate them [89]. In summary, incretin-based medications have pharmacological properties that enable them to inhibit lipotoxicity and thereby help to prevent lipotoxicity-dependent disorders, especially renal and cardiovascular complications [90].

5.3. Fatty Acid $\beta$-Oxidation. Fatty acid $\beta$-oxidation is a polyphasic enzymatic process in which FAs (long-chain acylCoA) are broken down to acetyl-CoA to produce energy [91]. This catabolic process occurs in mitochondria (as well as peroxisomes) to generate acetyl-CoA followed by nicotinamide adenine dinucleotide (NADH) and flavin adenine dinucleotide 2 (FADH2) which, in turn, enter the citric acid cycle and the mitochondrial electron transport chain, producing energy as adenosine triphosphate (ATP) [91]. This process consumes the FAs, thereby lowering serum FA levels and the overall body fat supply; it therefore plays a significant role in lipid homeostasis and determining the amount of fat in body tissues [91] as well as maintaining tissue energy balance [92]. Fatty acid $\beta$-oxidation is regulated at two major levels: transcriptional and posttranscriptional or allosteric [91]. While transcriptional regulation is performed by key proteins such as peroxisome proliferator-activated receptors (PPARs), sterol regulatory element-binding protein 1 (SREBP1), and peroxisome proliferator-activated receptor- $\gamma$ coactivator- $1 \alpha$ (PGC- $1 \alpha)$, allosteric control is carried out by the level of by-products produced which may negatively or positively affect the relevant metabolic enzymes [91]. Some evidence indicates that incretin-based medications can interact with these regulators [49, 93].

$\mathrm{Xu}$ and colleagues in 2016 demonstrated that exendin-4 induces fatty acid oxidation via a sirtuin 1- (SIRT-1-) dependent signaling pathway in 3T3L1 adipocytes [49]. They found that GLP-1 signaling improves oxidant capacity which, in turn, increases FA oxidation in cultured adipocytes [49]. A clinical study in 2019 demonstrated that liraglutide induces lipid oxidation and reduces adiposity in obese patients with T2DM [70]. Timper et al. in 2020 found that GLP-1R signaling promotes FA $\beta$-oxidation in cultured astrocytes which, in turn, improves lipid homeostasis and memory efficiency, suggesting that GLP-1 signaling is important to energy homeostasis and brain function through FA $\beta$-oxidation-dependent pathways [94]. Further studies have suggested that GLP-1 signaling exerts protective effects on energy homeostasis by promoting fatty acid oxida- tion [92]. GLP-1 can improve insulin sensitivity in hepatocytes by promoting hepatic fatty acid oxidation in both human and rat liver biopsies [95]. GLP-1 signaling induces an intrinsic signal to increase fatty acid oxidation and reduce insulin resistance in the diabetic state [96]. GLP-1 also interacts with genes involved in FA $\beta$-oxidation [93]. Recent evidence suggests that GLP-1 receptors expressed on cardiomyocytes provide cardioprotection through interactions that increase fatty acid beta-oxidation that, in turn, reduce epicardial adipose tissue thickness and improve cardiac function [93]. Taken together, this evidence demonstrates that GLP-1 signaling has a significant impact on fatty acid oxidation and may provide some of its beneficial effects as an insulin sensitizer and cardioprotective agent through modulation of FA $\beta$-oxidation.

5.4. Cholesterol Synthesis. Cholesterol is a lipid molecule which is synthesized by most eukaryotes [97]. It is involved in many important biologic activities, such as hormone synthesis and cell membrane formation [97]. However, in higher concentrations, it becomes a risk factor for cardiovascular disease [75]. Therefore, maintaining cholesterol in the physiologic range is critically important [75]. Recent evidence suggests that GLP-1 mimetics have a modulatory role on cholesterol homeostasis and may improve impaired cholesterol metabolism [98, 99].

Yao et al. in 2018 found that GLP-1 affects both cholesterol transport and synthesis, modulating cholesterol homeostasis via upregulation of ABCA-1and downregulation of miR-19b in isolated hepatocytes [98]. Kim et al. in 2020 demonstrated that GLP-1 improves brain function via improvement in cholesterol metabolism, suggesting that GLP-1's ability to regulate cerebral blood flow is dependent upon cholesterol homeostasis [99]. A study in 2018 demonstrated that the GLP-1 analogue liraglutide reduced circulating cholesterol levels in mice on a high fat diet [100]. Similar evidence from 2018 showed that GLP-1 receptor agonists reduce cholesterol synthesis by suppressing the HMG-CoA reductase (a key enzyme in cholesterol synthesis) and SREBP-1C [101]. These transcriptional effects of GLP-1 are cardioprotective by reducing atheroma plaque formation [101]. Clinical evidence from 2018 demonstrated that GLP1 administration reduces serum cholesterol levels and improves dyslipidemia by inhibition of HMG-CoA reductase in T2DM patients [102]. These findings strongly suggest that GLP-1 mimetics have a positive modulatory impact on cholesterol metabolism and, in turn, dyslipidemia and 


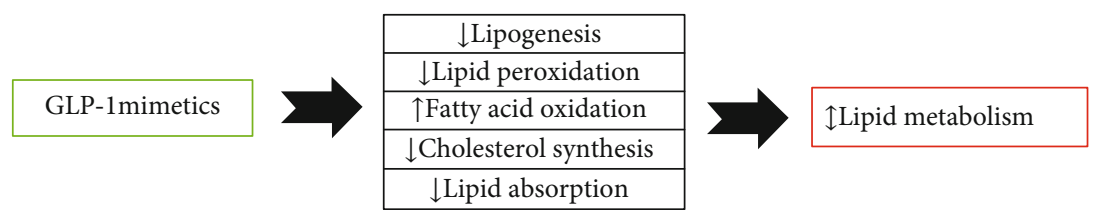

Figure 3: The different impacts of incretin-based medications on lipid metabolism.

atheroma plaque formation and therefore provide further protective lipid-modulating effects beyond their antidiabetic effects.

5.5. Lipid Absorption. Incretins are key regulators of intestinal lipid absorption [8]. GLP-1 has regulatory effects on satiety through the melanocortin 4 receptor- (MC4R-) mediated sympathetic system and by reducing lipid storage in both hepatic and adipose tissues [103]. Since GLP-1 is produced and secreted by intestinal L-cells and acts directly on the small intestine, it was suggested as likely that it affected intestinal lipid absorption [103]. Further studies showed that GLP-1 reduces postprandial chylomicron and TG circulatory levels by reducing intestinal lipid absorption [104]. GLP-1R agonists, such as exendin-4 (but not GIP), reduced intestinal lipid absorption and circulating levels of both triacylglycerol and ApoB-48 in an animal model on high fat diet [105]. GLP-1 also reduced intestinal lymph flow and TG absorption [106]. These findings suggest that endogenous GLP-1 regulates postprandial lipaemia through several pathways in addition to controlling lipid absorption [105].

\section{Conclusion}

Incretin-based medications are a novel class of antidiabetic drugs that provide potent modulatory effects on glucose metabolism. However, much evidence suggests that they also regulate lipid metabolism. Since most diabetic patients have some degree of dyslipidemia, the lipid-modulatory effects of incretins provide further pharmacological benefits for diabetic patients. Our review demonstrates that incretin-based therapies modulate lipid metabolism via at least five cellular pathways: lipogenesis and lipolysis, lipid peroxidation, lipid absorption, cholesterol biosynthesis, and fatty acid betaoxidation (Figure 3 ). We also present clinical evidence supporting the experimental findings. However, more experimental and clinical studies are still needed to further elucidate the molecular targets that are affected by incretinbased therapeutics to modulate lipid and lipoprotein metabolism. Moreover, evidence from lipoprotein tracer kinetic studies will pave the way towards elucidation of the impact of incretin-based agents on lipoprotein biogenesis and catabolism. There is also a lack of enough clinical evidence about the effects of incretin-based therapies on the risk of diabetic macrovascular and microvascular complications. Finally, the possible role of combining incretin-based drugs with common lipid-lowering agents in nondiabetic individuals remains open to question.

Taken together, this body of evidence suggests that incretin-based medications are effective antidiabetic thera- pies especially in patients with dyslipidemia, as they have the potential to normalize lipid metabolism in diabetes.

\section{Data Availability}

There is no raw data associated with this review.

\section{Conflicts of Interest}

The authors clearly declare that have no conflict of interest in this study.

\section{References}

[1] J. Divers, E. J. Mayer-Davis, J. M. Lawrence et al., “Trends in incidence of type 1 and type 2 diabetes among youths-selected counties and Indian reservations, United States, 2002-2015," Morbidity and Mortality Weekly Report, vol. 69, no. 6, pp. 161-165, 2020.

[2] N. E. López-Palau and J. M. Olais-Govea, "Mathematical model of blood glucose dynamics by emulating the pathophysiology of glucose metabolism in type 2 diabetes mellitus," Scientific Reports, vol. 10, no. 1, pp. 1-11, 2020.

[3] J. M. Forbes and M. E. Cooper, "Mechanisms of diabetic complications," Physiological Reviews, vol. 93, no. 1, pp. 137-188, 2013.

[4] R. H. Ritchie and E. D. Abel, "Basic mechanisms of diabetic heart disease," Circulation Research, vol. 126, no. 11, pp. 1501-1525, 2020.

[5] J. Barrera-Chimal and F. Jaisser, "Pathophysiologic mechanisms in diabetic kidney disease: a focus on current and future therapeutic targets," Diabetes, Obesity and Metabolism, vol. 22, pp. 16-31, 2020.

[6] S. Eid, K. M. Sas, S. F. Abcouwer et al., "New insights into the mechanisms of diabetic complications: role of lipids and lipid metabolism," Diabetologia, vol. 62, no. 9, pp. 1539-1549, 2019.

[7] H. Yaribeygi, T. Sathyapalan, and A. Sahebkar, "Molecular mechanisms by which GLP-1 RA and DPP-4i induce insulin sensitivity," Life sciences, vol. 234, article 116776, 2019.

[8] S. Farr, J. Taher, and K. Adeli, "Glucagon-like peptide-1 as a key regulator of lipid and lipoprotein metabolism in fasting and postprandial states," Cardiovascular \& Haematological Disorders-Drug Targets (Formerly Current Drug TargetsCardiovascular \& Hematological Disorders), vol. 14, no. 2, pp. 126-136, 2014.

[9] D. J. Drucker and M. A. Nauck, "The incretin system: glucagon-like peptide-1 receptor agonists and dipeptidyl peptidase- 4 inhibitors in type 2 diabetes," The Lancet, vol. 368, no. 9548, pp. 1696-1705, 2006.

[10] J. J. Meier, "GLP-1 receptor agonists for individualized treatment of type 2 diabetes mellitus," Nature Reviews Endocrinology, vol. 8, no. 12, pp. 728-742, 2012. 
[11] L. L. Baggio and D. J. Drucker, "Biology of incretins: GLP-1 and GIP," Gastroenterology, vol. 132, no. 6, pp. 2131-2157, 2007.

[12] K. A. Scott and T. H. Moran, "The GLP-1 agonist exendin-4 reduces food intake in nonhuman primates through changes in meal size," American Journal of Physiology-Regulatory, Integrative and Comparative Physiology, vol. 293, no. 3, pp. R983-R987, 2007.

[13] X. Ding, N. K. Saxena, S. Lin, N. Gupta, and F. A. Anania, "Exendin-4, a glucagon-like protein-1 (GLP-1) receptor agonist, reverses hepatic steatosis in ob/ob mice," Hepatology, vol. 43, no. 1, pp. 173-181, 2006.

[14] D. Wootten, J. Simms, C. Koole et al., "Modulation of the glucagon-like peptide-1 receptor signaling by naturally occurring and synthetic flavonoids," Journal of Pharmacology and Experimental Therapeutics, vol. 336, no. 2, pp. 540-550, 2011.

[15] M. Islam, "Insulinotropic effect of herbal drugs for management of diabetes mellitus: a congregational approach," Biosens J, vol. 5, no. 142, p. 2, 2016.

[16] S. Radbakhsh, S. L. Atkin, L. E. Simental-Mendia, and A. Sahebkar, "The role of incretins and incretin-based drugs in autoimmune diseases," International Immunopharmacology, vol. 98, p. 107845, 2021.

[17] S. Radbakhsh, T. Sathyapalan, M. Banach, and A. Sahebkar, "Incretins and microRNAs: interactions and physiological relevance," Pharmacological Research, p. 153, 2020.

[18] H. Yaribeygi, M. Maleki, S. L. Atkin, T. Jamialahmadi, and A. Sahebkar, "Impact of incretin-based therapies on adipokines and adiponectin," Journal of Diabetes Research, vol. 2021, 9 pages, 2021.

[19] H. Yaribeygi, M. Maleki, T. Sathyapalan, T. Jamialahmadi, and A. Sahebkar, "Anti-inflammatory potentials of incretinbased therapies used in the management of diabetes," Life Sciences, vol. 241, p. 117152, 2020.

[20] H. Yaribeygi, M. Maleki, T. Sathyapalan, T. Jamialahmadi, and A. Sahebkar, "Incretin-based therapies and reninangiotensin system: looking for new therapeutic potentials in the diabetic milieu," Life Sciences, p. 256, 2020.

[21] H. Yaribeygi, M. Maleki, T. Sathyapalan, T. Jamialahmadi, and A. Sahebkar, "Antioxidative potentials of incretin-based medications: a review of molecular mechanisms," Oxidative Medicine and Cellular Longevity, vol. 2021, 9 pages, 2021.

[22] H. Yaribeygi, A. Rashidy-Pour, S. L. Atkin, T. Jamialahmadi, and A. Sahebkar, "GLP-1 mimetics and cognition," Life Sciences, vol. 264, p. 118645, 2021.

[23] H. Yaribeygi, M. Ashrafizadeh, N. C. Henney, T. Sathyapalan, T. Jamialahmadi, and A. Sahebkar, "Neuromodulatory effects of anti-diabetes medications: a mechanistic review," Pharmacological Research, vol. 152, article 104611, 2020.

[24] A. D. Association, "2. Classification and diagnosis of diabetes: standards of medical care in diabetes-2018," Diabetes Care, vol. 41, Supplement 1, pp. S13-S27, 2018.

[25] B. Ahren, "DPP-4 inhibitors," Best Practice \& Research Clinical Endocrinology \& Metabolism, vol. 21, no. 4, pp. 517-533, 2007.

[26] F. R. Ponziani, S. Pecere, A. Gasbarrini, and V. Ojetti, "Physiology and pathophysiology of liver lipid metabolism," Expert review of gastroenterology \& hepatology, vol. 9, no. 8, pp. 1055-1067, 2015.

[27] J. E. Hall and M. E. Hall, Guyton and Hall Textbook of Medical Physiology e-Book, Elsevier Health Sciences, 2020.
[28] U. White and E. Ravussin, "Dynamics of adipose tissue turnover in human metabolic health and disease," Diabetologia, vol. 62, no. 1, pp. 17-23, 2019.

[29] M. Schoeler and R. Caesar, "Dietary lipids, gut microbiota and lipid metabolism," Reviews in Endocrine and Metabolic Disorders, vol. 20, no. 4, pp. 461-472, 2019.

[30] C.-W. Ko, J. Qu, D. D. Black, and P. Tso, "Regulation of intestinal lipid metabolism: current concepts and relevance to disease," Nature Reviews Gastroenterology \& Hepatology, vol. 17, no. 3, pp. 169-183, 2020.

[31] J. Long, C.-J. Zhang, N. Zhu et al., "Lipid metabolism and carcinogenesis, cancer development," American journal of cancer research, vol. 8, no. 5, pp. 778-791, 2018.

[32] L. Chen, X.-W. Chen, X. Huang, B.-L. Song, Y. Wang, and Y. Wang, "Regulation of glucose and lipid metabolism in health and disease," Science China Life Sciences, vol. 62, no. 11, pp. 1420-1458, 2019.

[33] F. Séguro, J. P. Rabès, D. Taraszkiewicz, J. B. Ruidavets, V. Bongard, and J. Ferrières, "Genetic diagnosis of familial hypercholesterolemia is associated with a premature and high coronary heart disease risk," Clinical cardiology, vol. 41, no. 3, pp. 385-391, 2018.

[34] G. Penno, A. Solini, G. Zoppini et al., "Hypertriglyceridemia is independently associated with renal, but not retinal complications in subjects with type 2 diabetes: a cross-sectional analysis of the Renal Insufficiency And Cardiovascular Events (RIACE) Italian multicenter study," PLoS One, vol. 10, no. 5, article e0125512, 2015.

[35] Y. Zhou, C. Wang, K. Shi, and X. Yin, "Relationship between dyslipidemia and diabetic retinopathy," Medicine, vol. 97, no. 36, p. e12283, 2018.

[36] C.-J. Jeng, Y.-T. Hsieh, C.-M. Yang, C.-H. Yang, C.-L. Lin, and I.-J. Wang, "Diabetic retinopathy in patients with dyslipidemia: development and progression," Ophthalmology Retina, vol. 2, no. 1, pp. 38-45, 2018.

[37] K. Haile and A. Timerga, "Dyslipidemia and its associated risk factors among adult type- 2 diabetic patients at Jimma University Medical Center, Jimma, Southwest Ethiopia," Diabetes, Metabolic Syndrome and Obesity: Targets and Therapy, vol. 13, pp. 4589-4597, 2020.

[38] M. G. Savelieff, B. C. Callaghan, and E. L. Feldman, "The emerging role of dyslipidemia in diabetic microvascular complications," Current Opinion in Endocrinology, Diabetes and Obesity, vol. 27, no. 2, pp. 115-123, 2020.

[39] V. G. Athyros, M. Doumas, K. P. Imprialos et al., "Diabetes and lipid metabolism," Hormones, vol. 17, no. 1, pp. 61-67, 2018.

[40] X. Li, L. Jiang, M. Yang, and Y.-w. Wu, "Expression of CTRP3, a novel adipokine, in rats at different pathogenic stages of type 2 diabetes mellitus and the impacts of GLP-1 receptor agonist on it," Journal of diabetes research, vol. 2014, 2014.

[41] T. Hosaka, M. Yoshida, N. Harada, H. Sakaue, T. Sakai, and Y. Nakaya, "Exendin-4, a GLP-1 receptor agonist, directly induces adiponectin expression through protein kinase A pathway and prevents inflammatory adipokine expression," Biochemical and biophysical research communications, vol. 390, no. 3, pp. 613-618, 2009.

[42] V. Sancho, M. V. Trigo, N. González, I. Valverde, W. J. Malaisse, and M. L. Villanueva-Peñacarrillo, "Effects of glucagon-like peptide-1 and exendins on kinase activity, 
glucose transport and lipid metabolism in adipocytes from normal and type-2 diabetic rats," Journal of molecular endocrinology, vol. 35, no. 1, pp. 27-38, 2005.

[43] M. Ahmadian, Y. Wang, and H. S. Sul, "Lipolysis in adipocytes," The international journal of biochemistry \& cell biology, vol. 42, no. 5, pp. 555-559, 2010.

[44] Y. Wang, J. Viscarra, S.-J. Kim, and H. S. Sul, "Transcriptional regulation of hepatic lipogenesis," Nature reviews Molecular cell biology, vol. 16, no. 11, pp. 678-689, 2015.

[45] G. Laliotis, I. Bizelis, and E. Rogdakis, "Comparative approach of the de novo fatty acid synthesis (lipogenesis) between ruminant and non ruminant mammalian species: from biochemical level to the main regulatory lipogenic genes," Current Genomics, vol. 11, no. 3, pp. 168-183, 2010.

[46] X. Zhao, D. Feng, Q. Wang et al., "Regulation of lipogenesis by cyclin-dependent kinase 8-mediated control of SREBP1," The Journal of clinical investigation, vol. 122, no. 7, pp. 2417-2427, 2012.

[47] S. Ducheix, J. Lobaccaro, P. Martin, and H. Guillou, "Liver X receptor: an oxysterol sensor and a major player in the control of lipogenesis," Chemistry and physics of lipids, vol. 164, no. 6, pp. 500-514, 2011.

[48] F. W. Sanders and J. L. Griffin, "De novo lipogenesis in the liver in health and disease: more than just a shunting yard for glucose," Biological Reviews, vol. 91, no. 2, pp. 452-468, 2016.

[49] F. Xu, B. Lin, X. Zheng et al., "GLP-1 receptor agonist promotes brown remodelling in mouse white adipose tissue through SIRT1," Diabetologia, vol. 59, no. 5, pp. 1059-1069, 2016.

[50] A. B. Engin, "What is lipotoxicity?," Obesity and lipotoxicity, pp. 197-220, 2017.

[51] R. E. Duncan, M. Ahmadian, K. Jaworski, E. Sarkadi-Nagy, and H. S. Sul, "Regulation of lipolysis in adipocytes," Annual Review of Nutrition, vol. 27, no. 1, pp. 79-101, 2007.

[52] H. M. Lando, M. Alattar, and A. P. Dua, "Elevated amylase and lipase levels in patients using glucagonlike peptide-1 receptor agonists or dipeptidyl-peptidase-4 inhibitors in the outpatient setting," Endocrine Practice, vol. 18, no. 4, pp. 472-477, 2012.

[53] Y. Saisho, "Incretin-based therapy and pancreatitis: accumulating evidence and unresolved questions," Annals of translational medicine, vol. 6, no. 7, p. 131, 2018.

[54] M. Almutairi, K. Gopal, A. A. Greenwell et al., "The GLP-1 receptor agonist liraglutide increases myocardial glucose oxidation rates via indirect mechanisms and mitigates experimental diabetic cardiomyopathy," Canadian Journal of Cardiology, vol. 37, no. 1, pp. 140-150, 2021.

[55] E. Tomas, V. Stanojevic, K. McManus et al., "GLP-1 (32-36) amide pentapeptide increases basal energy expenditure and inhibits weight gain in obese mice," Diabetes, vol. 64, no. 7, pp. 2409-2419, 2015.

[56] A. Veprik, R. B. Bakar, L. Vetterli et al., Acetyl-CoA-carboxylase 1 (ACC1) plays a critical role in glucagon and GLP1 secretion and controls whole body glucose homeostasis: session: the endocrine role of the gut: incretins and other hormones, European Association for the Study of Diabetes Virtual Meeting, 2019.

[57] J. Chen, H. Zhao, X. Ma et al., "GLP-1/GLP-1R signaling in regulation of adipocyte differentiation and lipogenesis," Cellular Physiology and Biochemistry, vol. 42, no. 3, pp. 11651176, 2017.
[58] S. Ben-Shlomo, I. Zvibel, M. Shnell et al., "Glucagon-like peptide-1 reduces hepatic lipogenesis via activation of AMP-activated protein kinase," Journal of hepatology, vol. 54, no. 6, pp. 1214-1223, 2011.

[59] E. T. Parlevliet, Y. Wang, J. J. Geerling et al., "GLP-1 receptor activation inhibits VLDL production and reverses hepatic steatosis by decreasing hepatic lipogenesis in high-fat-fed APOE* 3-Leiden mice," PLoS One, vol. 7, no. 11, article e49152, 2012.

[60] T. Ideta, Y. Shirakami, T. Miyazaki et al., "The dipeptidyl peptidase-4 inhibitor teneligliptin attenuates hepatic lipogenesis via AMPK activation in non-alcoholic fatty liver disease model mice," International journal of molecular sciences, vol. 16, no. 12, pp. 29207-29218, 2015.

[61] L. Zhang, M. Yang, H. Ren et al., "GLP-1 analogue prevents NAFLD in ApoE KO mice with diet and Acrp30 knockdown by inhibiting c-JNK," Liver International, vol. 33, no. 5 , pp. 794-804, 2013.

[62] J. Hsieh, K. E. Trajcevski, S. L. Farr et al., "Glucagon-like peptide 2 (GLP-2) stimulates postprandial chylomicron production and postabsorptive release of intestinal triglyceride storage pools via induction of nitric oxide signaling in male hamsters and mice," Endocrinology, vol. 156, no. 10, pp. 3538-3547, 2015.

[63] D. J. Cuthbertson, A. Irwin, C. J. Gardner et al., "Improved glycaemia correlates with liver fat reduction in obese, type 2 diabetes, patients given glucagon-like peptide-1 (GLP-1) receptor agonists," PLoS One, vol. 7, no. 12, article e50117, 2012.

[64] P. Zilleßen, J. Celner, A. Kretschmann, A. Pfeifer, K. Racké, and P. Mayer, "Metabolic role of dipeptidyl peptidase 4 (DPP4) in primary human (pre) adipocytes," Scientific Reports, vol. 6, no. 1, pp. 1-12, 2016.

[65] J. W. Day, N. Ottaway, J. T. Patterson et al., "A new glucagon and GLP-1 co-agonist eliminates obesity in rodents," Nature chemical biology, vol. 5, no. 10, pp. 749-757, 2009.

[66] C. Ruiz-Grande, C. Alarcón, E. Mérida, and I. Valverde, "Lipolytic action of glucagon-like peptides in isolated rat adipocytes," Peptides, vol. 13, no. 1, pp. 13-16, 1992.

[67] G. C. Yaney, V. N. Civelek, A.-M. Richard et al., "Glucagonlike peptide 1 stimulates lipolysis in clonal pancreatic betacells (HIT)," Diabetes, vol. 50, no. 1, pp. 56-62, 2001.

[68] M. Villanueva-Penacarrillo, L. Marquez, N. Gonzalez, M. Díaz-Miguel, and I. Valverde, "Effect of GLP-1 on lipid metabolism in human adipocytes," Hormone and Metabolic Research, vol. 33, no. 2, pp. 73-77, 2001.

[69] V. Patel, A. Joharapurkar, S. Kshirsagar et al., "Balanced coagonist of GLP-1 and glucagon receptors corrects dyslipidemia by improving FGF21 sensitivity in hamster model," Drug research, vol. 67, no. 12, pp. 730-736, 2017.

[70] C. Anholm, P. Kumarathurai, A. Samkani et al., "Effect of liraglutide on estimates of lipolysis and lipid oxidation in obese patients with stable coronary artery disease and newly diagnosed type 2 diabetes: a randomized trial," Diabetes, Obesity and Metabolism, vol. 21, no. 8, pp. 2012-2016, 2019.

[71] M. J. Pereira, K. Thombare, A. Sarsenbayeva et al., "Direct effects of glucagon on glucose uptake and lipolysis in human adipocytes," Molecular and cellular endocrinology, vol. 503, article 110696, 2020.

[72] V. Rago, D. de Rose, M. Santoro et al., "Human sperm express the receptor for glucagon-like peptide-1 (GLP-1), which 
affects sperm function and metabolism," Endocrinology, vol. 161, no. 4, p. bqaa031, 2020.

[73] V. Patel, A. Joharapurkar, S. Kshirsagar et al., "Central administration of coagonist of GLP-1 and glucagon receptors improves dyslipidemia," Biomedicine \& Pharmacotherapy, vol. 98, pp. 364-371, 2018.

[74] C. Wang, L. Li, S. Liu et al., "GLP-1 receptor agonist ameliorates obesity-induced chronic kidney injury via restoring renal metabolism homeostasis," PLoS One, vol. 13, no. 3, article e0193473, 2018.

[75] O. Castañer, X. Pintó, I. Subirana et al., "Remnant cholesterol, not LDL cholesterol, is associated with incident cardiovascular disease," Journal of the American College of Cardiology, vol. 76, no. 23, pp. 2712-2724, 2020.

[76] S. Camastra, B. Astiarraga, A. Tura et al., "Effect of exenatide on postprandial glucose fluxes, lipolysis, and B-cell function in non-diabetic, morbidly obese patients," Diabetes, Obesity and Metabolism, vol. 19, no. 3, pp. 412-420, 2017.

[77] F. Ursini and M. Maiorino, "Lipid peroxidation and ferroptosis: the role of GSH and GPx4," Free Radical Biology and Medicine, vol. 152, pp. 175-185, 2020.

[78] L. J. Marnett, "Lipid peroxidation-DNA damage by malondialdehyde," Mutation Research/Fundamental and Molecular Mechanisms of Mutagenesis, vol. 424, no. 1-2, pp. 83-95, 1999.

[79] S. Basu, D. De, H. D. Khanna, and A. Kumar, "Lipid peroxidation, DNA damage and total antioxidant status in neonatal hyperbilirubinemia," Journal of Perinatology, vol. 34, no. 7, pp. 519-523, 2014.

[80] V. Patel, A. Joharapurkar, N. Dhanesha et al., "Combination of omeprazole with GLP-1 agonist therapy improves insulin sensitivity and antioxidant activity in liver in type 1 diabetic mice," Pharmacological Reports, vol. 65, no. 4, pp. 927-936, 2013.

[81] M. C. Bunck, A. Cornér, B. Eliasson et al., "One-year treatment with exenatide vs. insulin glargine: effects on postprandial glycemia, lipid profiles, and oxidative stress," Atherosclerosis, vol. 212, no. 1, pp. 223-229, 2010.

[82] N. Lalitha, B. Sadashivaiah, T. R. Ramaprasad, and S. A. Singh, "Anti-hyperglycemic activity of myricetin, through inhibition of DPP-4 and enhanced GLP-1 levels, is attenuated by co-ingestion with lectin-rich protein," PLoS One, vol. 15, no. 4, article $\mathrm{e} 0231543,2020$.

[83] C. Huang, L. Yuan, and S. Cao, "Endogenous GLP-1 as a key self-defense molecule against lipotoxicity in pancreatic islets," International journal of molecular medicine, vol. 36, no. 1, pp. 173-185, 2015.

[84] M. J. Armstrong, D. Hull, K. Guo et al., "Glucagon-like peptide 1 decreases lipotoxicity in non-alcoholic steatohepatitis," Journal of hepatology, vol. 64, no. 2, pp. 399-408, 2016.

[85] J. Gu, Q. Wei, H. Zheng, X. Meng, J. Zhang, and D. Wang, "Exendin-4 promotes survival of mouse pancreatic $\beta$-cell line in lipotoxic conditions, through the extracellular signalrelated kinase 1/2 pathway," Journal of diabetes research, vol. 2016, 8 pages, 2016.

[86] E. Somm, S. A. Montandon, U. Loizides-Mangold et al., "The GLP-1R agonist liraglutide limits hepatic lipotoxicity and inflammatory response in mice fed a methionine-choline deficient diet," Translational Research, vol. 227, pp. 75-88, 2021.

[87] F. Liu, L. Gong, W. Qin, C. Cui, L. Chen, and M. Zhang, “Glucagon-like peptide 1 attenuates lipotoxicity-induced islet dys- function in $\mathrm{Apo}^{\mathrm{E}-{ }_{-}-}$Mice," Diabetes, Metabolic Syndrome and Obesity: Targets and Therapy, vol. Volume 13, pp. 27012709, 2020.

[88] X. Wang, J. Liu, C. Li et al., "Impaired secretion of active GLP-1 in patients with hypertriglyceridaemia: a novel lipotoxicity paradigm?," Diabetes/metabolism research and reviews, vol. 34, no. 2, article e2964, 2018.

[89] J.-H. Hong, D.-H. Kim, and M.-K. Lee, "Glucolipotoxicity and GLP-1 secretion," BMJ Open Diabetes Research and Care, vol. 9, no. 1, article e001905, 2021.

[90] K. Tomovic, J. Lazarevic, G. Kocic, M. Deljanin-Ilic, M. Anderluh, and A. Smelcerovic, "Mechanisms and pathways of anti-inflammatory activity of DPP-4 inhibitors in cardiovascular and renal protection," Medicinal research reviews, vol. 39, no. 1, pp. 404-422, 2019.

[91] Y. Ma, S. M. Temkin, A. M. Hawkridge et al., "Fatty acid oxidation: an emerging facet of metabolic transformation in cancer," Cancer letters, vol. 435, pp. 92-100, 2018.

[92] R. Al Batran, M. Almutairi, and J. R. Ussher, "Glucagon-like peptide-1 receptor mediated control of cardiac energy metabolism," Peptides, vol. 100, pp. 94-100, 2018.

[93] E. Dozio, E. Vianello, A. E. Malavazos et al., "Epicardial adipose tissue GLP-1 receptor is associated with genes involved in fatty acid oxidation and white-to-brown fat differentiation: a target to modulate cardiovascular risk?," International journal of cardiology, vol. 292, pp. 218-224, 2019.

[94] K. Timper, A. del Río-Martín, A. L. Cremer et al., "GLP-1 receptor signaling in astrocytes regulates fatty acid oxidation, mitochondrial integrity, and function," Cell metabolism, vol. 31, no. 6, pp. 1189-1205, 2020.

[95] G. Svegliati-Baroni, S. Saccomanno, C. Rychlicki et al., "Glucagon-like peptide-1 receptor activation stimulates hepatic lipid oxidation and restores hepatic signalling alteration induced by a high-fat diet in nonalcoholic steatohepatitis," Liver International, vol. 31, no. 9, pp. 1285-1297, 2011.

[96] R. Khound, J. Taher, C. Baker, K. Adeli, and Q. Su, "GLP-1 elicits an intrinsic gut-liver metabolic signal to ameliorate diet-induced VLDL overproduction and insulin resistance," Arteriosclerosis, thrombosis, and vascular biology, vol. 37, no. 12, pp. 2252-2259, 2017.

[97] J. Luo, H. Yang, and B.-L. Song, "Mechanisms and regulation of cholesterol homeostasis," Nature reviews Molecular cell biology, vol. 21, no. 4, pp. 225-245, 2020.

[98] Y. Yao, Q. Li, W. Wang, J. Zhang, P. Gao, and Y. Xu, “Glucagon-like peptide-1 modulates cholesterol homeostasis by suppressing the miR-19b-induced downregulation of ABCA1," Cellular Physiology and Biochemistry, vol. 50, no. 2, pp. 679-693, 2018.

[99] Y.-K. Kim and J. Song, "Potential of glucagon-like peptide 1 as a regulator of impaired cholesterol metabolism in the brain," Advances in Nutrition, vol. 11, no. 6, pp. 1686-1695, 2020.

[100] G. Rakipovski, B. Rolin, J. Nøhr et al., "The GLP-1 analogs liraglutide and semaglutide reduce atherosclerosis in $\mathrm{ApoE}^{-/-}$and $\mathrm{LDLr}^{-1-}$ mice by a mechanism that includes inflammatory pathways," Science, vol. 3, no. 6, pp. 844-857, 2018.

[101] V. Patel, A. Joharapurkar, S. Kshirsagar et al., "Coagonist of GLP-1 and glucagon decreases liver inflammation and atherosclerosis in dyslipidemic condition," Chemico-biological interactions, vol. 282, pp. 13-21, 2018. 
[102] Y. Hasegawa, M. Hori, T. Nakagami, M. Harada-Shiba, and Y. Uchigata, "Glucagon-like peptide-1 receptor agonists reduced the low-density lipoprotein cholesterol in Japanese patients with type 2 diabetes mellitus treated with statins," Journal of clinical lipidology, vol. 12, no. 1, pp. 62-69, 2018.

[103] G. M. Dallinga-Thie and M. Nieuwdorp, GLP1, an important regulator of intestinal lipid metabolism, American Heart Association, 2015.

[104] T. A. Lutz and E. Osto, "Glucagon-like peptide-1, glucagonlike peptide-2, and lipid metabolism," Current opinion in lipidology, vol. 27, no. 3, pp. 257-263, 2016.

[105] J. Hsieh, C. Longuet, C. Baker et al., "The glucagon-like peptide 1 receptor is essential for postprandial lipoprotein synthesis and secretion in hamsters and mice," Diabetologia, vol. 53, no. 3, pp. 552-561, 2010.

[106] X. Qin, H. Shen, M. Liu et al., "GLP-1 reduces intestinal lymph flow, triglyceride absorption, and apolipoprotein production in rats," Physiology, vol. 288, no. 5, pp. G943-G949, 2005.

[107] V. Sancho, M. V. Trigo, A. Martín-Duce et al., "Effect of GLP1 on D-glucose transport, lipolysis and lipogenesis in adipocytes of obese subjects," International journal of molecular medicine, vol. 17, no. 6, pp. 1133-1137, 2006.

[108] V. Sancho, B. Nuche, L. Arnés et al., "The action of GLP-1 and exendins upon glucose transport in normal human adipocytes, and on kinase activity as compared to morbidly obese patients," International journal of molecular medicine, vol. 19, no. 6, pp. 961-966, 2007. 